\title{
Algorithms for Integer Factorization Based on Counting Solutions of Various Modular Equations
}

\author{
Boris S. Verkhovsky \\ Computer Science Department, New Jersey Institute of Technology, Newark, USA \\ E-mail:verb73@gmail.com
}

Received September 20, 2011; revised October 27, 2011; accepted November 6, 2011

\begin{abstract}
This paper is a logical continuation of my recently-published paper. Security of modern communication based on RSA cryptographic protocols and their analogues is as crypto-immune as integer factorization (iFac) is difficult. In this paper are considered enhanced algorithms for the iFac that are faster than the algorithm proposed in the previous paper. Among these enhanced algorithms is the one that is based on the ability to count the number of integer solutions on quadratic and bi-quadratic modular equations. Therefore, the $i F a c$ complexity is at most as difficult as the problem of counting. Properties of various modular equations are provided and confirmed in numerous computer experiments. These properties are instrumental in the proposed factorization algorithms, which are numerically illustrated in several examples.
\end{abstract}

Keywords: RSA Cryptography, Integer Factorization, Modular Quadratic Equations, Modular Bi-Quadratic Equation, Equivalent Problems, Rabin Protocol

\section{Introduction and Problem Statement}

Security of modern communication based on RSA or Rabin cryptographic protocols and their analogues is as crypto-immune as difficult is the integer factorization (iFac) [1-3]. This paper is a continuation of the paper [4]. In that paper is considered a factorization algorithm of semi-prime $n=p q$ for two cases: where either both factors $p$ and $q$ are non-Blum primes i.e.,

$$
p=q=1(\bmod 4),
$$

or at least one factor is a non-Blum prime. In this paper an $i F a c$ algorithm is provided, which also works if both factors $p$ and $q$ are Blum primes, i.e.,

$$
p=q=3(\bmod 4) .
$$

The SQUAR-algorithm discussed in [4] is based on several properties (formulated as propositions and conjectures) of dual modular elliptic curves, where $b$ is a positive integer:

$$
y^{2}=x\left(x^{2}+b^{2}\right)(\bmod n)
$$

and

$$
y^{2}=x\left(x^{2}-b^{2}\right)(\bmod n) .
$$

Let us reiterate some of these properties and then consider their generalizations.
Let $p=q=1(\bmod 4) ; n=p q ;$ let $P(n, b)$ and $M(n, b)$ denote the number of points on elliptic curves (EC) (1.3) and (1.4) respectively.

For the sake of brevity, we call $P(n, b)$ and $M(n, b)$ the counts.

Conjecture 1.1: Consider $n=p q$, and let primes $p$ and $q$ satisfy (1.1);

if

$$
P(n, 1) \neq M(n, 1) ;
$$

then for every integer $b$

$$
P(n, b) \neq M(n, b) ;
$$

otherwise, for every integer $b$

$$
P(n, b)=M(n, b) .
$$

If $n$ is a prime and (1.5) holds, then for every $b$ also holds

$$
[P(n, b)+M(n, b)] / 2=n .
$$

Remark 1.1: Conjecture1.1 plays an important role in the design of the iFac described in [4]; further details are provided in the Appendix.

Proposition 1.2: If the factors $p$ and $q$ are congruent to 1 modulo $n=p q$, then the following identities hold for non-negative integers $m$ and $s$ : 
if

$$
|m-s| \bmod 4=2
$$

then

$$
P\left(n, 2^{m}\right)=M\left(n, 2^{s}\right) .
$$

Proposition 1.3: If the factors $p$ and $q$ are congruent to 1 modulo $n=p q, \quad b_{1} \neq b_{2}$, and

$$
P\left(n, b_{1}\right)=M\left(n, b_{2}\right),
$$

then

$$
M\left(n, b_{1}\right)=P\left(n, b_{2}\right) .
$$

The proposed iFac2 algorithm described below is less restrictive than the integer factorization SQUAR-algorithm described and analyzed in [4], because it is also applicable if both $p$ and $q$ satisfy (1.1).

Proposition 1.4: \{modular reduction-in-exponent\}: Consider elliptic curves

$$
y^{2}=x\left(x^{2}+b^{e}\right)(\bmod n)
$$

and

$$
y^{2}=x\left(x^{2}-b^{e}\right)(\bmod n)
$$

where $e \geq 4$; then for every integer $b>0$ and $e>0$ the following identities hold:

$$
\begin{gathered}
P\left(n, b^{e}\right)=P\left(n, b^{e \bmod 4}\right) ; \\
M\left(n, b^{e}\right)=M\left(n, b^{e \bmod 4}\right) .
\end{gathered}
$$

Proof \{by mathematical induction\}: Consider substitutions

$$
y:=Y b^{3} \bmod n \text { and } x:=X b^{2} \bmod n ;
$$

into (1.12). Then after cancellation of the same term $b^{6}$ in both parts of (1.12) we derive the EC

$$
Y^{2}=X\left(X^{2}+b^{e-4}\right)(\bmod n) .
$$

Repeating the substitutions (1.16) and cancellations of term $b^{6}$, we derive the proof of (1.14). Analogously we proceed with the proof of (1.15).

A generalized reduction-in-exponent can be formulated for a hyperelliptic curve $\{\mathrm{HEC}\}$.

Proposition 1.5: Consider HECs

$$
y^{r}=\left(x^{d} \pm x^{t} b^{e}\right)(\bmod n) ;
$$

and $Y^{r}=\left(X^{d} \pm X^{t} b^{e \bmod [(d-t) r / m]}\right)(\bmod n)$.

If $t<d$ and $\operatorname{gcd}(d, r)=m$, then for every integer $b>0$ both HECs have equal number of points.

Proof: after appropriate substitutions, the proof is analogous to the proof of Proposition 1.4 (details of the proof and an example are provided in the Appendix $\}$. Special case: if $m=1$ and $t=0$, then

$$
Y^{r}=\left(X^{d} \pm b^{e \bmod d r}\right)(\bmod n) \text {. }
$$

\section{2. iFac1 Algorithm Based on EC}

SQUAR-algorithm described in paper [4] requires consideration of a sequence of elliptic curves with control parameter $b$. Namely, for every $b=1,2,3,5, \cdots$ to count the number of points on each EC until four distinct counts are found; \{see Example 2.1 below .

In the following algorithm we need at most three distinct counts. Let $P_{i}:=P\left(n, b_{i}\right)$.

\section{The iFac1 algorithm:}

1) Compute $P_{1}, P_{2}, \cdots, P_{i} \neq P_{1}$ until two distinct integers are found;

2) if

$$
\begin{aligned}
& \operatorname{sign}\left(P_{1}-n\right)=\operatorname{sign}\left(P_{i}-n\right) \\
& p:=\operatorname{gcd}\left(P_{1}+P_{i}, n\right) ; \quad q=n / p ;
\end{aligned}
$$

then

else compute $w:=\operatorname{gcd}\left(P_{1}+P_{i}, n\right)$;

3) if $w>1$, then $p:=w$;

$$
\text { else find a } 3^{\text {rd }} \text { distinct count } P_{k} \text {; }
$$

4)

$$
p:=\operatorname{gcd}\left(P_{1}+P_{k}, n\right) ; q=n / p .
$$

Example 2.1: For semi-prime $n=6525401$, the sets $S_{1}, S_{2}, S_{3}$ and $S_{4}$ are as follows:

$$
\begin{aligned}
& S_{1}=\{b=1,7,11,17,29,31,41, \cdots ; L=7012681\} ; \\
& S_{2}=\{b=2,5,13,23,37, \cdots ; S=6055665\} ; \\
& S_{3}=\{b=3,19, \cdots ; A=6514053\} ; \\
& S_{4}=\{b=\underline{43}, 53, \cdots ; B=6519205\} .
\end{aligned}
$$

Therefore, the SQUAR-algorithm provided in [4] requires at least fifteen basic steps, because 43 is the fourteenth prime (2.5). Yet, since

$$
P_{1} \neq P_{2} ; \text { and } P_{1} \neq P_{3} \neq P_{2} \text {; }
$$

then the $1^{\text {st }}$ factor $:=\operatorname{gcd}\left(P_{1}+P_{3}, n\right)$.

Hence, instead of counting points

$$
P_{1}, P_{2}, P_{3}, P_{5}, P_{7}, \cdots, P_{43}
$$

in fifteen elliptic curves, we determine both factors of $n$ after three distinct counts.

\section{3. iFac1 Validation}

Definition 3.1: A pair of counts $\left\{P_{i}, P_{j}\right\}$ is called a resolventa if $\operatorname{gcd}\left(P_{i}+P_{j}, n\right)>1$.

If $w=1(2.1)$, then we need to compute the $3^{\text {rd }}$ distinct value \{see Example 2.1\}. However, if $w>1$, then we compute the $1^{\text {st }}$ factor, say, $p$, and then $q:=n / p$. The following proposition and examples provide explanations. 
Proposition 3.1: If primes $p$ and $q$ are selected randomly, then with probability greater than $2 / 3$ we can determine factors of semi-prime $n$ if we know only two distinct counts $P_{1}$ and $P_{i}$.

Proof: It is demonstrated in the paper [4] that if $p=q=1(\bmod 4)$, then there exist two positive integers $c<p$ and $d<q$, and four sets $S_{1}, S_{2}, S_{3}$ and $S_{4}$ such that for every $b$ the number of points on the elliptic curves (1.3) and (1.4) is equal either $A$ or $B$ or $L$ or $S$, where

$$
\begin{aligned}
& A:=(p+c)(q-d) ; \\
& B:=(p-c)(q+d) ; \\
& L:=(p+c)(q+d) ; \\
& S:=(p-c)(q-d) ;
\end{aligned}
$$

\{see Example2.1\}.

For instance, let's analyze

$$
\operatorname{gcd}(L+S, n)
$$

where

$$
L+S=2(n+c d) \quad \text { (3.2). }
$$

Let's find under what conditions $p$ divides $L+S$ : suppose that

$$
n+c d=p h,
$$

where $h$ is an integer. Then (3.5) implies

$$
\begin{gathered}
\text { that } \quad(n+c d) \bmod p=p h \bmod p=0 ; \\
\text { and } \quad c d \bmod p=0 .
\end{gathered}
$$

Since $c<p$, therefore, $p$ must divide $d$.

Hence, if $\quad c<p \leq d<q$, and $p \mid d$,

then we can find factors $p$ and $q$ after considerations of only two distinct counts $P_{1}$ and $P_{i}$. Although this case is possible \{see Example 3.2\}, for large primes $p$ and $q$ it is highly improbable.

Analogously, we proceed with an analysis of $\operatorname{gcd}(A+B, n)$.

Example 3.1: Consider $n=9037729$;

and

$$
\text { EC } y^{2}=x^{3}+x(\bmod n) \text {. }
$$

If $P_{1}=A=8894593 ; P_{i}=B=9176905 ;$ then compute

$$
w:=\operatorname{gcd}(A+B, n)=1 .
$$

Since $w=1$, it means that we cannot find the factors of $n$ because the combination $\{A, B\}$ is not a resolventa $\{$ see Table 3.1 $\}$. Yet, after we find the third distinct value $P_{k}=L=9342205$; the factorization is accomplished:

$$
p=3361 \text { and } q=2689 \text {. }
$$

Example 3.2: $\{$ Highly improbable case $\}$ : Consider $n=24853$. Let's verify that, if we know any two counts, we can find $p$ and $q$. There are six cases to consider:
1). $P_{1}=A ; P_{i}=B$;
2). $P_{1}=A ; P_{i}=L$
3). $P_{1}=A ; P_{i}=S$;
4). $P_{1}=B ; P_{i}=L$;
5). $P_{1}=B ; P_{i}=S$;
6). $P_{1}=L ; P_{i}=S$;

Table 3.1. Sums and greatest common divisors.

\begin{tabular}{ccc}
\hline Sums $\boldsymbol{X}+\boldsymbol{Y}$ & $\boldsymbol{g c d}(\boldsymbol{X}+\boldsymbol{Y}, \boldsymbol{n})$ & Resolventas \\
\hline$A+B=2(n-c d)$ & $\geq 1$ & No \\
$A+L=2(p+c) \boldsymbol{q}$ & $q$ & Yes \\
$A+S=2 \boldsymbol{p}(q-d)$ & $p$ & Yes \\
$B+L=2 \boldsymbol{p}(q+d)$ & $p$ & Yes \\
$B+S=2(p-c) \boldsymbol{q}$ & $q$ & Yes \\
$L+S=2(n+c d)$ & $\geq 1$ & No \\
\hline
\end{tabular}

where $A=17385 ; B=31161 ; L=35685$; and $S=15181$. Then for each of these combinations we find a factor of $n$. Indeed,

$$
\begin{aligned}
& \operatorname{gcd}(A+B, n)=29 ; \quad \operatorname{gcd}(B+L, n)=857 ; \\
& \operatorname{gcd}(B+S, n)=29 ; \quad \operatorname{gcd}(A+L, n)=29 ; \\
& \operatorname{gcd}(A+S, n)=857 ; \quad \operatorname{gcd}(L+S, n)=29 .
\end{aligned}
$$

Although such case is possible, it is highly improbable if $p$ and $q$ are randomly selected.

Example 3.3: Consider $n=8405801$ and EC (3.9). Compute $P_{1}=8387409 ; P_{2}=P_{1} ; P_{3}=8995597 ;$ and $w:=\operatorname{gcd}\left(P_{1}+P_{3}, n\right)=2801$.

Because $w>1$, therefore

$$
p:=w \text { and } q:=n / p=3001 \text {. }
$$

In general, every combination $\{A, L\}$, or $\{A, S\}$, or $\{B, L\}$ or $\{B, S\}$ has a common factor. Hence, if $w=1$,

then $\operatorname{gcd}\left(P_{1}+P_{k}, n\right)>1$,

otherwise

$$
\operatorname{gcd}\left(P_{1}+P_{i}, n\right)>1 \text {. }
$$

Since $n$ is a semi-prime, then in each of these cases we compute a factor of $n$. For instance, if

$$
\begin{aligned}
& P_{1}=A \text { and } P_{i}=L, \\
& \text { then } \quad A+L=(p+c) q ; \\
& \text { and } \quad \operatorname{gcd}[(p+c) q, n]=q .
\end{aligned}
$$

For more details see Table A2.

Although the $i F a c 1$ algorithm is computationally simpler than the SQUAR algorithm, we can further simplify the iFac algorithm via application of other modular equations.

\section{Modular Quadratic and Bi-quadratic Equations}

In this section are considered properties of quadratic, bi-quadratic modular equations and equations with $m \geq 3$, where the moduli are prime or semi-prime.

Proposition 4.1: Consider a modular quadratic equation (MQE)

$$
y^{2}=x^{2}-b(\bmod n)
$$


let $G(n, b)$ denote the number of integer pairs $(x, y)$ \{called points on quadratic curve (4.1)\} that satisfy (4.1); if $n$ is a prime, then for every non-zero $b$ co-prime with $n$

$$
G(n, b)=n-1 \text {; }
$$

if $n$ is a semi-prime and $n=p q$, then for every non-zero $b$ co-prime with $n$

$$
G(p q, b)=(p-1)(q-1) .
$$

Proof is provided in the Appendix.

Conjecture 4.2: Consider a modular equation $V(p, m, b)$ :

$$
y^{2}=x^{2 m}-b(\bmod p)
$$

where $p$ is a prime; let $G(p, m, b)$ denote the number of points on (4.3);

if (4.3) is either a quadratic or bi-quadratic equation (i.e., if $m=1$ or $m=2$ ), and $p \bmod 4=3$, then

$$
G(p, m, b)=p-1 \text {. }
$$

if $m=1$ and $p \bmod 4=1$, then (4.4) holds.

Table 4.1. Values of $G(p, m, b)$.

\begin{tabular}{cccc}
\hline$p \bmod 4$ & $m=1$ & $m=2$ & $m \geq 3:$ if $\operatorname{gcd}(m, p-1)=1$ \\
\hline$p \bmod 4=1$ & $p-1$ & $\neq p-1$ & $p-1$ \\
$p \bmod 4=3$ & $p-1$ & $p-1$ & $p-1$ \\
\hline
\end{tabular}

Conjecture 4.3: Consider a modular equation $V(n, m, b)$ : let $b>0$;

$$
y^{2}=x^{2 m}-b(\bmod n) ;
$$

and let $G(n, m, b)$ denote the number of points on (4.5); if both factors $p$ and $q$ are primes, and if (4.5) is either a quadratic or bi-quadratic equation (i.e., if $m=1$ or $m=2$ ), then for every $b>0$

$$
G(p q, m, b)=(p-1)(q-1) ;
$$

if an odd prime $m$ is co-prime with $(p-1)(q-1)$, then

\begin{tabular}{|c|c|c|c|}
\hline & $m=1$ & $m=2$ & $m>3$; if $\operatorname{gcd}[m, \varphi(n)]=1$ \\
\hline$p=q(\bmod 4)=1$ & $\varphi(n)$ & $\neq \varphi(n)$ & $\varphi(n)$ \\
\hline$p=q(\bmod 4)=3$ & $\varphi(n)$ & $\varphi(n)$ & $\varphi(n)$ \\
\hline$p q \bmod 4=3$ & $\varphi(n)$ & $\neq \varphi(n)$ & $\varphi(n)$ \\
\hline
\end{tabular}
for every $b$ and $m$ each co-prime with $\varphi(n)$

$$
G(n, m, b)=(p-1)(q-1)=\varphi(n) .
$$

Here $\varphi(n)$ is called the Euler totient function.

Table 4.2. Values of $G(p q, m, b)$.
Numerous computer experiments for $m=2,3,5,7$ confirmed Conjecture 4.3 Thirty six examples in Table 4.3 demonstrate the correctness of the Conjecture 4.3 for $m=1,2,3$, and 5 . In italics are shown the cases, where $\operatorname{gcd}[m, \varphi(n)]>1$, i.e., where (4.7) does not hold.

Table 4.3. Values of $G(p q, m, b) ; m=1,2,3,5$.

\begin{tabular}{cccc}
\hline & $m=1$ & $m=2$ & $m \geq 3 ;$ if $\operatorname{gcd}[m, \varphi(n)]=1$ \\
\hline 65,85, & 48,64, & 36,28 & $\boldsymbol{m}=\mathbf{3}: 32,64,224$ \\
377 & $336 \boldsymbol{o} \boldsymbol{m}$ & 324 & $\boldsymbol{m}=\mathbf{5}: 48,64,336$ \\
77,161 & 60,132 & 60,132 & $\boldsymbol{m}=\mathbf{3}: 140,308,20$ \\
209 & $180 \boldsymbol{o} \boldsymbol{1}$ & $180 \boldsymbol{o}$ & $\boldsymbol{m}=\mathbf{5}: 12,132,36$ \\
55,95 & 40,72 & 20,36, & $\boldsymbol{m}=\mathbf{3 :} 40,8,160$ \\
187 & $160 \boldsymbol{o}$ & 140 & $\boldsymbol{m}=\mathbf{5}: 8,72,32$ \\
\hline
\end{tabular}

\{see also Table 6.1 and 6.2 below\}.

The iFac algorithm described below is based on Proposition 4.1. This algorithm is computationally efficient if there exists an efficient procedure (an oracle) that counts the points on either the MQE $(m=1)$ or bi-quadratic equation $(m=2)(4.5)$.

Definition 4.1: $\left\{\right.$ equivalence\}: Problem $A_{1}$ is equivalent to problem $A_{2}$ if their time complexities satisfy the inequality $T\left(A_{1}\right) \leq T\left(A_{2}\right)$.

Definition 4.2: strong equivalence\}: Problems $A_{1}$ and $A_{2}$ are strongly equivalent if their time complexities $T_{1}$ and $T_{2}$ satisfy $\Theta\left(T_{1}\right)=\Theta\left(T_{2}\right)$.

Tables 6.1 and 6.2 illustrate Conjecture 4.2 and Conjecture 4.3 .

\section{5. iFac2 Algorithm}

Conjecture 4.3 can be applied to design an iFac2 algorithm. As it implied from the following discussion, this algorithm is more efficient than the SQUAR-algorithm proposed in [4]. Yet, for the seemingly simple iFac2 algorithm we need to know how to efficiently count the number of points $G(n, m, b)$ on modular Equation (4.5) for $m=1$ or $m=2$.

\section{The algorithm}

1) Select $b=m=1$; compute $G(n)$ for $V(n, 1,1)$ (4.5) and (4.6);

2) Compute

$$
R:=n-G(n)+1
$$

3) Solve quadratic equation

$$
z^{2}-R z+n=0
$$

suppose $z_{1}$ and $z_{2}$ are its roots;

4) $\{$ Integer factors $p$ and $q\}$ :

$$
p:=z_{1} \text { and } q:=z_{2} \text {. }
$$

Therefore, the $i F a c 2$ problem is equivalent to the problem of counting points on the MQE (4.1). 
Remark 5.1: It is well-known that, if $n$ is a semi-prime and if we know the value of Euler totient function $\varphi(n)$ (4.7), then we can find the factors of $n$. The Conjecture 4.3 is the framework that allows us to compute $\varphi(n)$. then

Example 5.1: Let $n=98,743,069$;

$$
G(n)=98,723,196 \text {; }
$$

and

$$
R:=n-G(n)+1=19874 .
$$

The quadratic equation

$$
z^{2}-19874 z+98743069=0 ;
$$

has two roots:

\begin{tabular}{|c|c|c|c|c|c|c|c|c|c|c|}
\hline$p$ & $m=2$ & $m=3$ & $m=5$ & $m=7$ & * & $p$ & $m=2$ & $m=3$ & $m=5$ & $m=7$ \\
\hline 59 & 58 & 58 & 58 & 58 & $*$ & 2011 & 2010 & 2186 & 2162 & 2010 \\
\hline 101 & 98 & 100 & 92 & 100 & $*$ & 2017 & 1998 & 2084 & 2016 & 2284 \\
\hline 1777 & 1854 & 1748 & 1776 & 1776 & $*$ & 99923 & 99992 & 99992 & 99992 & 99992 \\
\hline 1913 & 1998 & 1912 & 1912 & 1912 & $*$ & 99991 & 99990 & 101102 & 101102 & 99990 \\
\hline
\end{tabular}

$$
z_{1,2}=9937 \pm 30
$$

Table 6.1. $V(p, m, 1): y^{2}=x^{2 m}+1(\bmod p)$.

Table 6.2. $V(p, m, 1)$ for $10^{6}<p<10^{7}$.

\begin{tabular}{cccccc}
\hline $\boldsymbol{p}$ & $\boldsymbol{m}=\mathbf{1}$ & $\boldsymbol{m}=\mathbf{2}$ & $\boldsymbol{m}=\mathbf{3}$ & $\boldsymbol{m}=\mathbf{5}$ & $\boldsymbol{m}=\mathbf{7}$ \\
\hline $\mathbf{2 , 6 9 6 , 5 2 7}$ & 2696526 & 2696526 & $\mathbf{2 6 8 9 9 5 8}$ & 2696526 & 2701694 \\
$\mathbf{5 , 2 6 4 , 6 4 7}$ & 5264646 & 5264646 & $\mathbf{5 2 7 3 7 2 6}$ & 5264646 & 5264646 \\
$\mathbf{6 , 8 7 8 , 4 0 7}$ & 6878406 & 6878406 & $\mathbf{6 8 7 5 9 1 8}$ & 6878406 & 6878406 \\
\hline
\end{tabular}

Hence,

$$
p:=z_{1}=9967 \text { and } q:=z_{2}=9907 .
$$

\section{Properties of Modular Equations for $m>1$ : Computer Experiments}

Table 6.1 describes results of computer experiments for various primes $p$ and

$$
y^{2}=x^{2 m}+1(\bmod p) .
$$

Remark 6.1: In Tables 6.1 and 6.2 in italic are indicated cases where $G(m, p) \neq p-1$ if $\operatorname{gcd}(m, p-1) \neq 1$. Notice that since

$$
101 \equiv 1777 \equiv 1913 \equiv 2017 \equiv 1(\bmod 4),
$$

the bi-quadratic modular equations do not have exactly $p-1$ points.

\section{7. iFac2 Algorithm Validation}

From Conjecture 4.3, the number of points $G(n, m)$ on modular Equation (4.5) is equal

$$
G(p q, m)=(p-1)(q-1) .
$$

If there is a computationally efficient algorithm that computes $G(n, 1)$ or $G(n, 2)$, then it implies that for $m \leq 2$

$$
p+q=n+1-G(n, m) \text {. }
$$

Therefore, by the Vieta theorem, $p$ and $q$ are the roots of quadratic equation

$$
z^{2}-[n+1-G(n, m)] z+n=0 .
$$

Q.E.D

\section{Conclusions}

Several factorization algorithms were described and analyzed in [4] and in this paper \{see Table 8.1 . It is obvious that modular Equation (4.5) can be used for the iFac 2 only if either $m=1$ or $m=2$. From the paper it follows that the complexity of integer factorization is at most as difficult as the problem of counting how many solutions have modular Diophantine equations. Therefore, the problem of counting points on the MQE is equivalent with the iFac2 problem.

Table 8.1. Algorithms \& residues modulo 4.

\begin{tabular}{ccc}
\hline Algorithm & Case1 & Case2 \\
\hline \multirow{2}{*}{ SQUAR } & Four ECs: & Not \\
& $(8.1)-(8.8) ;[4]$ & applicable \\
iFac1 & Three ECs: & Not \\
& $(2.1)-(2.6)$ & applicable \\
iFac2 & One MQE: & One MQE: \\
& $(5.1)-(5.4)$ & $(5.1)-(5.4)$ \\
\hline
\end{tabular}

Case1: $p=q=1(\bmod 4)$ or $(p+q) \bmod 4=0$;

Case2: $p=q=3(\bmod 4)$. 


\section{Acknowledgements}

I express my appreciation to A. Joux, D. Kanevsky, A. Koval, R. Rubino and to reviewers for suggestions that improved this paper.

\section{References}

[1] R. L. Rivest, A. Shamir and L. M. Adleman, "A Method for Obtaining Digital Signature and Public-Key Cryptosystems," Communications of ACM, Vol. 21, No. 2, 1978, pp. 120-126. doi:10.1145/359340.359342

[2] H. Elkamchouchi, K. Elshenawy and H. Shaban, "Extended RSA Cryptosystem and Digital Signature Schemes in the Domain of Gaussian Integers," Proceedings of the 8th International Conference on Communication Systems, Singapore City, Vol. 1, 25-28 November 2002, pp. 91-95.

[3] M. O. Rabin, "Digitalized Signatures and Public Key Functions as Intractable as Factorization," Technical Report MIT/LCS/TR-212, MIT Laboratory for Computer Science, Cambridge, January 1979.

[4] Boris S. Verkhovsky, "Integer Factorization of Semi- primes Based on Analysis of a Sequence of Modular Elliptic Equations," International Journal of Communications, Network and System Sciences, Vol. 4, No. 10, 2011, pp. 609-615. doi:10.4236/ijcns.2011.410073

[5] C. Pomerance, "The Quadratic Sieve Factoring Algorithm," Advances in Cryptology, Proceedings of Eurocrypt'84, LNCS, Vol. 209, Springer-Verlag, Berlin, 1985, pp. 169182.

[6] R. Schoof, "Counting Points on Elliptic Curves over Finite Fields," Journal de Theorie des Nombres de Bordeaux, Vol. 7, No. 1, 1995, pp. 219-254. doi:10.5802/jtnb. 142

[7] K. Rubin and A. Silverberg, "Ranks of Elliptic Curves," Bulletin (New Series) of the American Mathematical Society, Vol. 39, No. 4, 2002, pp. 455-474.

[8] L. Dewaghe, "Remarks on the Schoof-Elkies-Atkin Algorithm," Mathematics of Computation, Vol. 67, No. 223, 1998 , pp. $1247-1252$ doi:10.1090/S0025-5718-98-00962-4

[9] C. F. Gauss, "Theoria Residuorum Biquadraticorum," 2nd Edition, Chelsea Publishing Company, New York, 1965, pp. 534-586. 


\section{Appendix}

\section{A1. Proof of Proposition 4.1}

Consider MQE:

$$
y^{2}=x^{2}-b(\bmod n) .
$$

Proposition 4.1: If $n$ is a prime, then the number of points with non-negative $x$ and $y$ on quadratic curve $Q(n)$ is equal $n-1$; if $n=p q$, then $Q(p q)=(p-1)(q-1)$.

Proof: Consider an integer parameter $t$ on interval $[1, n-1]$. The modular multiplicative inverse of $t$ exists if and only if $\operatorname{gcd}(t, n)=1$.

Consider $v=\left(t+t^{-1} b\right)(n+1) / 2(\bmod n)$;

and

$$
w=\left(t^{-1} b-t\right)(n+1) / 2(\bmod n) .
$$

If $n$ is a prime, then there are $n-1$ integers that are co-prime with $n$; if $n$ is a semi-prime and $n=p q$, then there are $(p-1)(q-1)$ integers that are co-prime with $n$. If $n$ is odd, then $(n+1) / 2$ is an integer; therefore both $v$ and $w$ are integers.

It is easy to verify that for every $\underline{t}$ there is a unique pair $\{v, w\}$ that satisfies (A.1). Therefore, we proved that (A.1) has at least $n-1$ solutions for $n$ prime and has at least $(p-1)(q-1)$ if $n=p q$. Let us show that there are no other solutions.

Let assume that there exists a solution $(g, h)$ that is distinct from every pair in (A.2). First of all,

$g^{2}-h^{2}=b(\bmod n)$, which implies that, if $1 \leq b \leq n-1$, then neither $(g-h) \bmod n=0$;

nor

$$
(g+h) \bmod n=0 \text {. }
$$

Consider an integer

$$
u:=(g-h) \bmod n \neq 0 ;
$$

where $1 \leq u \leq n-1$;

then

$$
g+h=u^{-1} b \bmod n
$$

Thus,

$$
g=\left(u+u^{-1} b\right) \times 2^{-1} \bmod n
$$

and

$$
h=\left(u^{-1} b-u\right) \times 2^{-1} \bmod n .
$$

If $n$ is odd, then modular inverse of 2 exists and

$$
2^{-1} \bmod n=(n+1) / 2 \bmod n .
$$

Hence, the solution $(g, h)$ has the same parametric representation as $(v, w)$, if $u=t$. The contradiction proves the
Proposition 4.1.

Example A1: Consider Q(17):

$$
y^{2}=x^{2}-2(\bmod 17) \text {. }
$$

There are sixteen points on $\mathrm{Q}(17)$ :

$$
( \pm 6,0) ;(0, \pm 7) ;( \pm 1, \pm 4) ;( \pm 2, \pm 6) ;( \pm 7, \pm 8) \text {. }
$$

\section{A2. Complexity Analysis}

There are several algorithms that count points on elliptic and hyper-elliptic curves. If some of these algorithms can be applied for counting points on quadratic or bi-quadratic modular equations with the same time complexities, then the Schoof-Elkies-Atkin (SEA) algorithm is currently the best known algorithm that counts points on a modular cubic curve with expected running time $O\left(\log ^{4} p\right)$ [5-7]. Therefore, if, for instance, $p$ is of order $O\left(2^{1024}\right)=O\left(10^{307}\right)$, then

$$
O\left(\log ^{4} p\right)=O\left(2^{40}\right)=O\left(10^{12}\right) .
$$

Because the SEA algorithm does not work if $a=1$ and $b=0$ [8], consider a modular equation $y^{2}=x^{2}+b^{2}(\bmod p)$ with $|b| \neq 0$ and an algorithm with complexity $O\left(\log ^{s} p\right)$ that counts points on this curve. Since there are algorithms with complexity $O\left(\log ^{8} p\right)$ that count points for every elliptic curve, therefore $s \leq 8$. Thus

$$
O\left(\log ^{s} p\right)=O\left(2^{10 s}\right)=O\left(10^{3 s}\right) \text {. }
$$

This implies that in the worst case the problem can be solved with complexity $O\left(10^{24}\right)$.

\section{A3. Proof of Proposition 1.5}

Consider hyperelliptic curves (HECs)

$$
y^{r}=\left(x^{d}+x^{t} b^{e}\right)(\bmod n)
$$

and

$$
Y^{r}=\left(X^{d}+X^{t} b^{e \bmod [(d-t) r / m]}\right)(\bmod n) .
$$

If $0 \leq t<d$ and $\operatorname{gcd}(d, r)=m$, then for every positive integer $b$ both HECs have equal number of points.

Proof: Consider substitutions

$$
x:=X b^{w} ; y:=Y b^{z} ;
$$

into Equation (A.7); then we derive

$$
Y^{r} b^{r z}=\left(X^{d} b^{d w} \pm X^{t} b^{t w+e}\right)(\bmod n) .
$$

Now let us find such integers $w$ and $z$, for which the following equation holds 


$$
r z=d w(\bmod \varphi(n)) .
$$

The case is simplified if $t \ll \varphi(n)$ and $d \ll \varphi(n)$.

If $\operatorname{gcd}(r, d)=m$; then $w=r / m$ and $z=d / m$.

Hence, $d r \leq r t+e m$, i.e. $(d-t) r / m \leq e$.

Therefore, after cancellation of equal terms in both sides of the modular Equation (A.10), we derive a HEC

$$
Y^{r}=\left(X^{d} \pm X^{t} b^{e-(d-t) r / m}\right)(\bmod n) .
$$

Example A3: Let consider HEC

$$
y^{6}=\left(x^{15}+x^{11} b^{1777}\right)(\bmod 1913) ;
$$

then HEC $Y^{6}=\left(X^{15}+b X^{11}\right)(\bmod 1913)$ has the same number of points as (A.12).

Table A1. \# of EC and sequence in which $A, B, L$ and $S$ are computed; here $S<A<B<L$.

\begin{tabular}{ccccccc}
\hline $\boldsymbol{n}$ & $\boldsymbol{P}_{\mathbf{1}} ; \boldsymbol{b}=\mathbf{1}$ & $\boldsymbol{P}_{\mathbf{2}} ; \boldsymbol{b}$ & $\boldsymbol{P}_{\mathbf{3}} ; \boldsymbol{b}$ & $\boldsymbol{P}_{\mathbf{4}} ; \boldsymbol{b}$ & $\boldsymbol{p}$ & $\boldsymbol{q}$ \\
\hline $\mathbf{3 4 3 4 9 4 1}$ & $\boldsymbol{B}=3537485 ; 1$ & $\boldsymbol{L}=3633945 ; 2$ & $A=3328341 ; 5$ & $S=3239993 ;-5$ & 1933 & 1777 \\
$\mathbf{4 0 1 6 8 1 3}$ & $\boldsymbol{B}=4034637 ; 1$ & $\boldsymbol{S}=3748057 ; 2$ & $L=4294809 ; 5$ & $A=3989749 ;-5$ & 2113 & 1901 \\
$\mathbf{4 6 4 7 1 6 9}$ & $\boldsymbol{A}=4552177 ; 1$ & $\boldsymbol{B}=4731865 ; 11$ & $\boldsymbol{S}=4330189 ; 13$ & $L=4974445 ; 17$ & 3121 & 1489 \\
$\mathbf{4 9 1 5 1 8 9}$ & $\boldsymbol{S}=4557869 ; 1$ & $\boldsymbol{A}=4836777 ; 2$ & $B=4980665 ; 7$ & $L=5285445 ;-7$ & 1489 & 3301 \\
$\mathbf{6 2 9 5 0 5 7}$ & $\boldsymbol{B}=6394801 ; 1$ & $\boldsymbol{L}=6509965 ; 5$ & $S=6082957 ; 7$ & $A=6192505 ; 11$ & 2017 & 3121 \\
$\mathbf{9 0 3 7 7 2 9}$ & $\boldsymbol{A}=8894593 ; 1$ & $\boldsymbol{S}=8737213 ; 11$ & $B=9176905 ; 13$ & $L=9342205 ; 19$ & 3361 & 2689 \\
$\mathbf{9 9 0 6 4 3 3}$ & $\boldsymbol{L}=10181817 ; 1$ & $\boldsymbol{S}=9633073 ; 2$ & $\boldsymbol{A}=9717861 ; 5$ & $B=10092981 ; 7$ & 5021 & 1973 \\
\hline
\end{tabular}

Remark A1: In five of seven experiments, the very first two counts $\{B, L\} ;\{B, S\} ;\{S, A\} ;\{B, L\} ;$ and $\{A, S\}$ are resolventas, i.e. they provide a factor of $n$ : $p:=\operatorname{gcd}\left(P_{1}+P_{2}, n\right)$. 